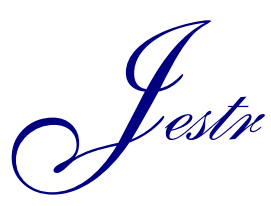

\title{
Patch antenna using Biocompatible Insulation layer in Wireless On-Body sensor applications
}

\author{
T. Anita Jones Mary*, Sugumar Durai and Shanon Mistry
}

Department of Electronics and Communication, Karunya University, Coimbatore 641114, Tamil Nadu, India

Received 4 October 2018; Accepted 8 January 2019

\begin{abstract}
On-body remote gadgets are low-power gadgets that go underneath the Medical Body Area Networks (MBANs). These remote or wireless gadgets give an inexpensive approach to watch the patients in the therapeutic field. The primary issue of the remote gadgets is the antenna, that is, the design of the antenna. This work introduces a patch antenna that can be utilized for remote on-body sensors to gauge the pulse of the patients. The antenna configuration approach includes a transmitting patch that has slots and a loop around the patch which is rectangular. The antenna is designed on a flexible substrate made of Polyimide material. With the goal to build the execution of the antenna, air substrate is utilized to such an extent that the antenna prerequisites are fulfilled. The proposed antenna is then reproduced on a human body phantom whose electrical properties like permittivity and conductivity are roughly the same to that of a human body. A biocompatible insulation layer is encompassed at the base of the antenna to diminish the Specific Absorption Rate (SAR) impact. The antenna parameters like return loss, VSWR, bandwidth and SAR esteems have been examined in this work.
\end{abstract}

Keywords: Department of Electronics and Communication, Karunya University, Coimbatore 641114, Tamil Nadu, India

\section{Introduction}

As of late, there has been an intensifying interest and advancement in Wireless Communication Systems [1-4]. With the mounting headway of these remote correspondence innovations, the focus is given now to the investigation of Wireless Body Area Network (WBAN) by a few scientists. The advancement of WBAN prompts the Medical Body Area Network (MBAN). MBAN assumes a fundamental job in the therapeutic field as it needs persistent observing of patients. These MBAN gadgets might be embedded inside the body utilized far from the body or might be surface mounted on the body in an unbending or firm position contingent on the necessities. This innovation has hauled worldwide consideration since it could give persistent and inconspicuous checking for communal healthcare factors like temperature, pulse, ECG, EEG, etc [5-6]. The gadgets utilized for therapeutic reason ought to be scaled down, lightweight and perfect, as they should be fused inside or on the body. It is fundamental for these gadgets to maintain a trustworthy correspondence with agreeable execution. In MBAN frameworks, one of the principal worries for the gadgets is the antenna design. This is on account of as expressed by the Link Budget investigation the framework imperatives have an immediate association with antenna's execution [7]. In this way, the antenna body will strikingly affect the impedance and resonant frequency of the antenna. Also, since there is significant power retention by the collection of people, the antenna has a tendency to experience a reduction in the radiation pattern. Likewise, the radiation example of the antenna can be distorted, all things

*E-mail address: anitajones@karunya.edu ISSN: $1791-2377 @ 2019$ Eastern Macedonia and Thrace Institute of Technology. All rights reserved. doi:10.25103/jestr.121.11 considered, [4], [8]. Therefore, because of this, it can prompt an expansion in mistakes identified with the transmission or much of the time a slight loss in correspondence connectivity. [9-12].

The antenna is required to have lack of care towards the closeness impact of the body with a specific end goal to show exact and stable execution. To shield the body from the hurtful impacts of radiations, the antenna is required to have the least radiation toward the human body. In [13-17], trial examinations have been done by the researchers to enhance the execution of the antennas. By and large unidirectional antennas are favoured for on-body correspondences to lessen the body presentation to electromagnetic (EM) field radiation. Since the patch antenna is unidirectional in nature, it is suitable to use in this work. Studies demonstrate that by utilizing a PEC plane close to the posterior of the antenna, its parameters like gain and productivity can be made strides. Certain engrossing materials when utilized on the rear of the antenna can decrease backward radiation [10],[18]. Anyway because of complex assembling forms, unbending structures and vast cumbersome sizes, these past works can't be utilized for satellite applications. Keeping in mind the end goal to defeat these issues, numerous specialists have brought up focus around low profile flexible antennas like material antennas. All the same, the antennas subject to discontinuities in view of a bending of the body [19-21]. These antennas are additionally reliant on different elements like dampness, material, producing process and substrate material [22].

In this paper, a patch antenna with the flexible substrate is proposed as on-body antenna for medicinal applications and its performance parameters are investigated. Besides a comparative report on biocompatible insulation layer with a various thickness which is presented between the body and the antenna is made. The performance parameters (return 
loss, VSWR, radiation pattern, gain) of the antenna are estimated and its performance is analyzed. Likewise, the consequences for antenna performance because of the closeness of the human body are considered.

\section{Antenna Approach and Design}

\subsection{Antenna Geometry}

The antenna approach is delineated in Fig.1. A remote sensor is utilized to quantify the pulse of the human body. It is connected to the frontal thorax specifically. The pulse that is estimated will then be sent to a close by the remote gadget. The general volume of the sensor is $70 \mathrm{~mm} \times 25 \mathrm{~mm}$ $\times 2 \mathrm{~mm}$.

\begin{tabular}{|c|c|c|}
\hline Flexible PCB & \multicolumn{2}{|c|}{ Antenna pattern } \\
\hline \multicolumn{2}{|r|}{ Air } & \multirow{2}{*}{$\begin{array}{c}\text { Via hole } \\
\begin{array}{c}\text { RF control } \\
\text { section }\end{array}\end{array}$} \\
\hline $\begin{array}{c}\text { Power control } \\
\text { section }\end{array}$ & $\begin{array}{l}\text { Logic control } \\
\text { section }\end{array}$ & \\
\hline \multicolumn{3}{|c|}{ Flexible PCB } \\
\hline \multicolumn{3}{|c|}{ Flexible battery } \\
\hline \multicolumn{3}{|c|}{ Electrode (Flexible PCB) } \\
\hline \multicolumn{3}{|c|}{ Human Body } \\
\hline
\end{tabular}

Fig. 1. Side view of sensor

In this work, the sensor comprises of three components are sensor cover and a framework module. The frame module consists of a flexible substrate, flexible battery and anodes. The flexible battery will be in charge of giving power to the whole framework. The pulse will be estimated by the flexible anodes which will be in close contact with the human body. These cathodes will be set at the base side of the sensor. The proposed antenna is planned on the highest side of the sensor with the goal that it tends to be utilized as a front of the sensor too. Since the structure of the antenna is little and smaller, it presents numerous confinements in the outlining of antenna and the general adaptability.

In spite of these limitations, the proposed work has various points of interest. Since the antenna is planned on the best side of the sensor, there is no need of additional space for a cover layer. This gives an advantage in keeping up the minimal size of the sensor. The adaptability of the sensor can be kept up as the substrate comprises of two polyimide layers of $0.15 \mathrm{~mm}$ thickness. The general idea of the sensor is portrayed in Fig 1. For on-body correspondences, patch antenna are broadly utilized since it can keep the radiation from going towards the body. The conductivity of the human body and its permittivity will have a lesser impact on the gadget.

In this work, the area of the patch antenna is at the highest point of the sensor. The structure of the antenna has an air substrate in the centre which contributes to expanding the effectiveness.

\subsection{Antenna Configuration and Design methodology}

To design the antenna for wireless on body sensor application, the battery, module and anodes are excluded. The structure of the antenna at that point comprises two layers of polyimide of $0.15 \mathrm{~mm}$ and an air layer in the centre which is of $1 \mathrm{~mm}$ in thickness. The flexible sheet comprises polyimide alongside thin layers of copper. These layers are utilized for the emanating top and additionally the base of the antenna. The aggregate measurements or volume of the whole proposed antenna structure are $70 \times 25 \times 1.3 \mathrm{~mm}^{3}$. The polyimide film has loss tangent of 0.005 , the permittivity of 2.7 and a thickness of $0.15 \mathrm{~mm}$

The most well-known prototypes for the design analysis of microstrip patch antennas are Transmission line model, Cavity model and Full wave model. In this effort, the transmission line model is utilized for the examination of the patch antenna in light of its straightforwardness and simplicity of estimations. The design is basically founded on the exact conditions overseeing the transmission line model. The subtle elements of measurement are portrayed in Fig 2.

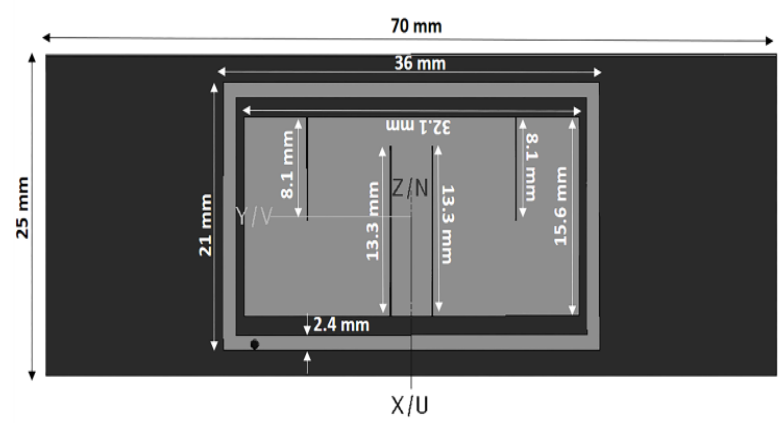

Fig. 2. Details of the proposed design

The antenna comprises of a rectangular patch. This patch is encompassed by a loop which is likewise rectangular. The capacity of the loop line in this work is to adjust the sustaining and it prompts the current to the transmitting patch by methods for EM coupling between the loop and the rectangular patch. The coupling impact can be balanced by fluctuating the separation between the loop and the patch. The proposed antenna is sustained by a $100 \Omega$ differential feeding line. The rectangular patch additionally comprises of vertical slots. These slots help in decreasing the size of the antenna. By lessening the size, the fabrication cost can be diminished all things considered. The simulations were done in CADFEKO 7.0 software. This software is preferred over other software's as this has 3D platform based electromagnetic simulation software. The operating frequency in this work is $2.36 \mathrm{GHz}$ to $2.39 \mathrm{GHz}$. To get wanted execution in this recurrence extension, the antenna can be balanced as needs be by changing the parameters like the width of the patch and the length of the slots.

\section{Antennas in Vicinity of Human Body}

\subsection{Modelling of the human body}

Since the utilization of wearable antennas requires it to work in close nearness to that of the person's body, we have to survey the parameter changes in the antenna when working in such a situation. Keeping in mind the end goal to study and measure the EM wave spread around and inside the body, testing the conduct of wearable antennas in the real human body may not be the best choice (and it may not be moral). The immediate contact between the tissue and the EM fields amid the estimations can have numerous undesirable results to human medicinal services. For the reenactment of wearable antennas, it is attractive to utilize numerical phantoms that reproduce human tissues. In this 
work, the proposed antenna is set on the chest of the patient. In such a manner, three-layered human chest involving skin, fat and muscle, displayed with dielectric parameters as appeared in Table 1. The organized model is portrayed in Fig 3 alongside the antenna. The multi-layered structure has measurements of $300 \mathrm{~mm} \times 300 \mathrm{~mm} \times 31.8 \mathrm{~mm}$.

Table 1. Parameters of human body model

\begin{tabular}{l|l|l|l}
\hline Tissues & $\begin{array}{l}\text { Relative } \\
\text { permittivity }\end{array}$ & $\begin{array}{l}\text { Conductivity } \\
(\mathbf{S} / \mathbf{m})\end{array}$ & $\begin{array}{l}\text { Thickness } \\
(\mathbf{m m})\end{array}$ \\
\hline Skin & 38.0 & 1.46 & 1.30 \\
Fat & 10.8 & 0.27 & 10.5 \\
Muscle & 52.7 & 1.74 & 20 \\
\hline
\end{tabular}

The simulation setup of the human model according to the parameters is depicted in Fig 3.

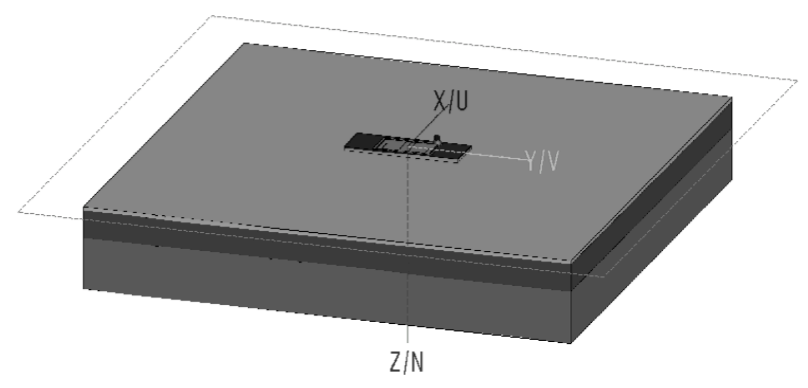

Fig.3 Simulation of Proposed antenna on Human body model

\subsection{Specific Absorption Rate (SAR)}

To discover the amount of electromagnetic vitality caught by the tissues in the body, assessment is done through a metric called Specific Absorption Rate (SAR) . Clearly, when the body is presented to the Radio Frequency (RF) waves, it ingests the radiation. What's more, the rate at which the human body ingests this radiation is known as the SAR. One strategy for figuring SAR is averaging it over the entire body while the other technique is to gauge over a little example volume (ordinarily 10 gram or 1 gram of tissue). SAR can be calculated as

$$
S A R=\int\left(\frac{\sigma(r)\left|E(r)^{2}\right|}{\rho(r)}\right) d r
$$

Where $\sigma(r)$ - Electrical Conductivity of the example, ERMS Electric Field, and $\rho(\mathrm{r})$ - Sample Density. As per the IEEE C95.1-1999 standard, $1.6 \mathrm{~W} / \mathrm{Kg}$ is the most extreme admissible incentive for SAR.

\subsection{Biocompatible Layer}

A biomaterial is a material which is intended to connect with organic frameworks. It is a material which is well disposed to the human body and does not have any lethal impacts. To keep the retention of radiation by the human body tissues and also towards the human body, the antenna is encompassed by a biocompatible insulation layer. The insulation layer thickness and sort assume a critical job on the SAR. Here in this work, the antenna structure with measurements $(70 \times 25 \times 1.3 \mathrm{~mm} 3)$ is encompassed by Teflona biocompatible insulation layer on the base layer. The insulation layer thickness and sort assume a critical job on the SAR esteem. The permittivity and loss tangent of Teflon are 2.1 and 0.0028 separately.

\section{Results and Discussion}

The proposed antenna was designed and simulated utilizing CADFEKO 7.0 software. The simulations were done for an arrangement of frequencies starting from $2.30 \mathrm{GHz}$ to 2.42 $\mathrm{GHz}$ to analyze the working of the antenna. The impact on the execution of the antenna because of the closeness of a human body phantom is researched. The patch antenna in the proposed work resonates at $2.374 \mathrm{GHz}$ without closeness of human body. The antenna has a return loss of - $10 \mathrm{~dB}$ without the nearness of the human body as demonstrated in Fig. 4.

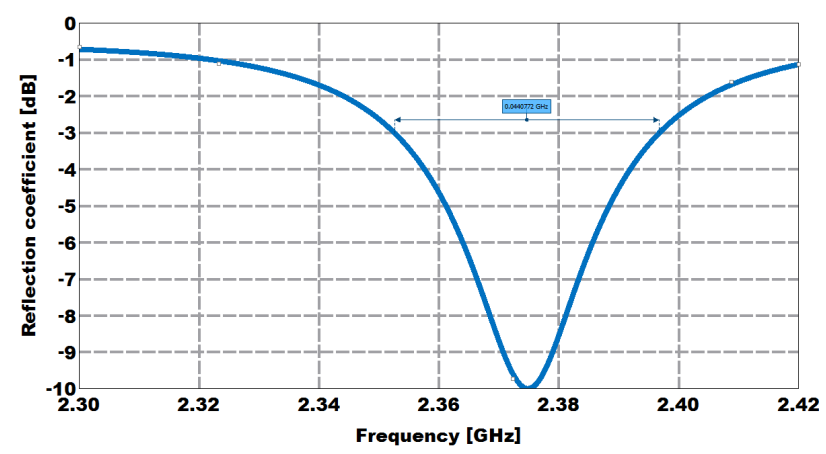

Fig.4 Return loss of proposed antenna

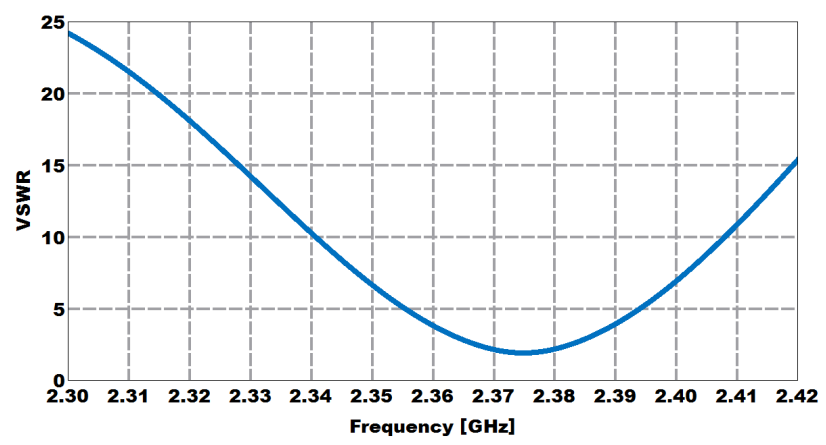

Fig. 5.VSWR of the antenna

Likewise, it covers a bandwidth of $44.07 \mathrm{MHz}$ The $3 \mathrm{~dB}$ bandwidth of the antenna covers the 2.36 to $2.39 \mathrm{GHz}$ extent which is our required operating frequency. The VSWR which as per the definition is the proportion of greatest voltage to the base voltage in the standing wave is estimated as 1.08 which can be found in the Fig.5. The antenna has a gain of $-3.90 \mathrm{dBi}$. In this work, the gain is seen to be less because of the small dimensions of the antenna.

At the point when put in close contiguity a human body phantom, the antenna demonstrates a return loss of - 15.62 $\mathrm{dB}$. Since there is high electrical permittivity of the human body, there is a slight drift in resonant frequency. The frequency encounters a shift from $2.374 \mathrm{GHz}$ without human body nearness to $2.376 \mathrm{GHz}$ on the body demonstrate.

Likewise, it can be delineated from the outcomes that the reproduced $3-\mathrm{dB}$ bandwidth on the phantom is observed to be $41.3 \mathrm{MHz}$ This bandwidth fulfils the MBAN band application prerequisites. The peak gain of the antenna in the nearness of the human body is $-9.82 \mathrm{dBi}$. The examination of the reflection coefficient of the antenna with the human body demonstrate and without is appeared in Fig.7 


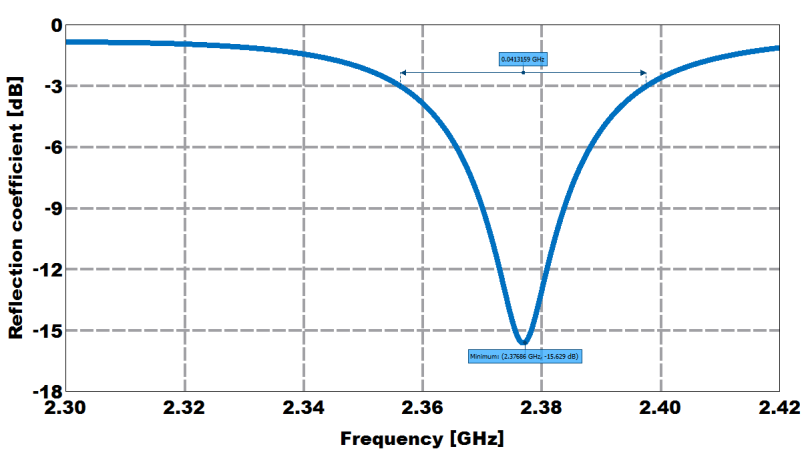

Fig. 6 Return loss of antenna with human body model

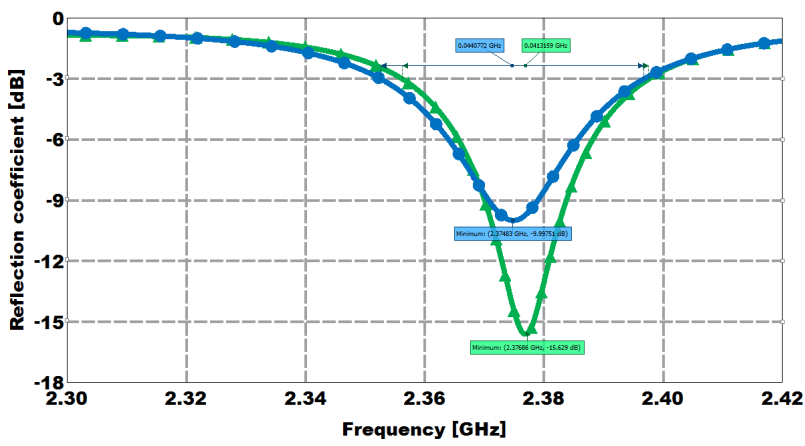

Fig. 7. Comparison of return loss of antenna with and without human body model

The SAR estimation of the antenna is $0.300 \mathrm{~W} / \mathrm{kg}$ and it is not as much as the most extreme admissible estimation of $1.6 \mathrm{~W} / \mathrm{kg}$. The correlation of results when the antenna is set close to a human body model and far from a human body display is indicated in Table 2 .

Table 2. Comparison of results

\begin{tabular}{c|c|c}
\hline Parameter & $\begin{array}{c}\text { Without human } \\
\text { body model }\end{array}$ & $\begin{array}{c}\text { With human } \\
\text { body model }\end{array}$ \\
\hline $\begin{array}{c}\text { Reflection } \\
\text { coefficient }\end{array}$ & $-10 \mathrm{~dB}$ & $-15.62 \mathrm{~dB}$ \\
Gain & $-3.90 \mathrm{~dB}$ & $-9.82 \mathrm{~dB}$ \\
SAR & - & $0.300 \mathrm{~W} / \mathrm{kg}$ \\
\hline
\end{tabular}

To diminish the SAR value for the patient's wellbeing the antenna is protected with a biocompatible material. The correlation of antenna parameters with insulation layer is delineated in Table 3 .

Table 3. Comparison of results with insulation layer

\begin{tabular}{c|c|c}
\hline $\begin{array}{c}\text { Thickness of insulation } \\
\text { (mm) }\end{array}$ & $\begin{array}{c}\text { Reflection } \\
\text { coefficient }\end{array}$ & SAR \\
\hline 0.5 & 8.25 & 0.079 \\
1 & -6.94 & 0.024 \\
1.3 & -4.05 & 0.006 \\
\hline
\end{tabular}

\section{Conclusion}

This methodology researches a patch-type slot antenna for a scaled down and little as well as the flexible remote sensor. The antenna is a patch antenna with slots and a loop encompassing the patch. Air is utilized as the center substrate. This is to adjust the exchange off the connection between antenna size and bandwidth by utilizing a multilayer substrate of various materials. The antenna execution in close contiguity a human body phantom is displayed and approved through recreated estimations. The antenna demonstrates a reflection coefficient of $-10 \mathrm{~dB}$ in free space while $-15.62 \mathrm{~dB}$ in the vicinity of a human body apparition. The $3 \mathrm{~dB}$ bandwidth of the antenna still covers the MBAN band and gives a bandwidth of $41.31 \mathrm{MHz}$ With a specific end goal to diminish the SAR esteem, a bio-good insulation layer is covered at the base of the proposed antenna. The diverse thickness of the insulation layer is connected to with the end goal that the SAR lessens up to 0.079 which is an exceptionally negligible esteem. Along these lines, the proposed antenna could be utilized little and flexible biosensor frameworks. Despite the fact that the antenna configuration process was painstakingly contemplated amid this work, the gain of the antenna is significantly low. Consequently, any gain improvement methods can be connected for future work.

This is an Open Access article distributed under the terms of the Creative Commons Attribution License

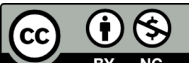

\section{References}

1. Biswas, Akash, Akib Jayed Islam, Abdullah Al-Faruk, and Sadman Shahriar Alam. "Design and performance analysis of a microstrip line-fed on-body matched flexible UWB antenna for biomedical applications." In Electrical, Computer and Communication Engineering (ECCE), International Conference on, pp. 181-185. IEEE, 2017.

2. H.-B. Li, K.-I. Takizawa, B. Zhen, and R. Kohno, "Body area network and its standardization at IEEE 802.15.MBAN,"in Proc. 16th IST Mobile Wireless Commun. Summit, Jul. 1-5, 2007, pp. 15.

3. Kiourti A, Psathas KA, Nikita KS. Implantable and ingestible medical devices with wireless telemetry functionalities: A review of current status and challenges. Bioelectromagnetics. 2014 Jan;35(1):1-5.

4. Chaturvedi D, Raghavan S. Circular quarter-mode SIW antenna for WBAN application. IETE Journal of Research. 2018 Jul 4;64(4), pp.482-8.

5. Jung YH, Qiu Y, Lee S, Shih TY, Xu Y, Xu R, Lee J, Schendel AA, Lin W, Williams JC, Behdad N. A compact parylene-coated WLAN flexible antenna for implantable electronics. IEEE Antennas and Wireless Propagation Letters. 2016,(6) pp.1382-5.
6. Kim, Sang-Gyu, Hee-Jo Lee, and Jong-Gwan Yook. "A biomolecular sensing platform using RF active system." J. Electromagn. Eng. Sci. 12, no. 4 (2012): 227-233.

7. Lo, Benny, and Guang-Zhong Yang. "Body sensor networksresearch challenges and opportunities." (2007): 26-32.

8. Conway, Gareth A., and William G. Scanlon. "Antennas for overbody-surface communication at $2.45 \mathrm{GHz}$." IEEE Transactions on Antennas and Propagation 57, no. 4 (2009): 844-855.

9. See, Terence SP, and Zhi Ning Chen. "Experimental characterization of UWB antennas for on-body communications." IEEE Transactions on Antennas and Propagation 57, no. 4 (2009): 866-874.

10. Klemm, Maciej, István Z. Kovcs, Gert F. Pedersen, and Gerhard Troster. "Novel small-size directional antenna for UWB WBAN/WPAN applications." IEEE Transactions on Antennas and Propagation 53, no. 12 (2005): 3884-3896.

11. Wong, Kin-Lu, and Chun-I. Lin. "Characteristics of a $2.4-\mathrm{GHz}$ compact shorted patch antenna in close proximity to a lossy medium." Microwave and Optical Technology Letters 45, no. 6 (2005): 480-483. 
12. Karimabadi, Sara Sadat, and Amir Reza Attari. "Gain enhancement of small size UWB antenna for wireless body area network applications." In Electrical Engineering (ICEE), 2010 18th Iranian Conference on, pp. 50-53. IEEE, 2010.

13. Haga, Nozomi, Kazuyuki Saito, Masaharu Takahashi, and Koichi Ito. "Characteristics of cavity slot antenna for body-area networks." IEEE Transactions on Antennas and Propagation 57, no. 4 (2009): 837-843.

14. Yarovoy, A. G., R. Pugliese, J. H. Zijderveld, and L. P. Ligthart. "Antenna development for UWB impulse radio." In Microwave Conference, 2004. 34th European, vol. 3, pp. 1257-1260. IEEE, 2004.

15. Alomainy, Akram, Yang Hao, and Frank Pasveer. "Numerical and experimental evaluation of a compact sensor antenna for healthcare devices." IEEE Transactions on Biomedical Circuits and Systems 1, no. 4 (2007): 242-249.

16. Chahat, Nacer, Maxim Zhadobov, Ronan Sauleau, and Koichi Ito. "A compact UWB antenna for on-body applications." IEEE Transactions on Antennas and Propagation 59, no. 4 (2011): $1123-$ 1131

17. Cho, Namjun, Taehwan Roh, Joonsung Bae, and Hoi-Jun Yoo. "A planar MICS band antenna combined with a body channel communication electrode for body sensor network." IEEE
Transactions on Microwave Theory and Techniques 57, no. 10 (2009): 2515-2522.

18. Kim, Ui-Sheon, and Jae-Hoon Choi. "An implantable antenna for wireless body area network application." Journal of electromagnetic engineering and science 10, no. 4 (2010): 206-211.

19. Kim, Seongjin, Kyeol Kwon, and Jaehoon Choi. "Design of a miniaturized high-isolation diversity antenna for wearable WBAN applications." Journal of electromagnetic engineering and science 13, no. 1 (2013): 28-33.

20. Kennedy, Timothy F., Patrick W. Fink, Andrew W. Chu, Nathan J. Champagne, Gregory Y. Lin, and Michael A. Khayat. "Body-worn E-textile antennas: the good, the low-mass, and the conformal." IEEE Transactions on Antennas and Propagation 57, no. 4 (2009): 910-918.

21. Hirvonen, Mervi, Christian Böhme, Daniel Severac, and Mickael Maman. "On-body propagation performance with textile antennas at $867 \mathrm{MHz}$." IEEE Transactions on Antennas and Propagation 61, no. 4 (2013): 2195-2199.

22. Bai, Qiang, and Richard Langley. "Crumpling of PIFA textile antenna." IEEE Transactions on Antennas and Propagation 60, no. 1 (2012): 63-70 